\title{
DESEMPENHO GENOTÍPICO DE LINHAGENS DE ARROZ DE TERRAS ALTAS UTILIZANDO METODOLOGIA DE MODELOS MISTOS (1)
}

\author{
VANDERLEY BORGES $\left({ }^{2}\right)$; ANTONIO ALVES SOARES $\left({ }^{(*}\right)$; MOIZÉS SOUZA REIS $\left({ }^{4}\right)$; \\ MARCOS DEON VILELA RESENDE (5); VANDA MARIA OLIVEIRA CORNÉLIO (4); \\ NATÁLIA ALVES LEITE ( $\left.{ }^{6}\right)$; ANTONIO RODRIGUES VIEIRA $\left(^{4}\right)$
}

\begin{abstract}
RESUMO
Avaliou-se o desempenho de genótipos de arroz de terras altas utilizando metodologia de modelos mistos. Foram utilizados dados de produtividade de grãos dos ensaios de valor de cultivo e uso (VCU) do programa cooperativo de melhoramento de arroz de terras altas, desenvolvido em Minas Gerais, no período de 1997/1998 a 2007 / 2008. Realizou-se análise de deviance para verificar a significância dos efeitos de genótipos e suas interações com locais e anos, obtendo-se a estabilidade e a adaptabilidade genotípicas, com os métodos da média harmônica dos valores genotípicos. Os efeitos de genótipos, genótipos x locais e genótipos $\mathrm{x}$ anos foram estatisticamente significativos $(p \leq 0,05)$. As linhagens e cultivares mais estáveis foram: Curinga-3, CNA 8983, Guarani, BRSMG Caravera e CNA 8824. Com maior adaptabilidade, estabilidade e produtividade de grãos, simultaneamente, destacaram-se a BRSMG Caravera, Curinga-3, MG 1089, MG 1097 e CNA 8436. A BRSMG Caravera, entre todas as cultivares e linhagens avaliadas de 1997 a 2008, foi a de melhor desempenho pela MHPRVG, ou seja, maior estabilidade, adaptabilidade e produtividade de grãos, simultaneamente, proporcionando aos orizicultores de Minas Gerais alta segurança no seu cultivo.
\end{abstract}

Palavras-chave: Oryza sativa, REML, BLUP, estabilidade genotípica, adaptabilidade.

\section{ABSTRACT \\ GENOTYPIC PERFOMANCE OF LINES OF THE UPLAND RICE USING MIXED MODEL}

The objective of this study was to evaluate the performance of genotypes of upland rice using mixed models methodology. Were evaluated data from yield tests of value for cultivation and use (VCU) from the cooperative improvement program of upland rice, developed in Minas Gerais during 1997/1998 to 2007/2008. Analysis of deviance was done to test the significance of the effects of genotypes and their interactions with sites and years, being obtained the stability and adaptability genotypic using the methods of the harmonic mean of genotypic values. The effects of genotypes, genotype $x$ sites and genotype $x$ year were statistically significant $(p \leq 0.05)$. The lines and cultivars Curinga-3, CNA 8983, Guarani, BRSMG Caravera and CNA 8824 were more stable, with greater adaptability, stability and yield, while the highlights were the BRSMG Caravera, Curinga-3, MG 1089, MG 1097 and CNA 8436. The BRSMG Caravera among all the cultivars and lines evaluated from 1997 to 2008, showed the best performance by MHPRVG, ie, greater stability, adaptability and yield while providing rice farmers from Minas Gerais a better crop.

Key words: Oryza sativa, REML/BLUP, adaptability, genotypic stability.

(1) Recebido para publicação em 13 de outubro de 2009 e aceito em 12 de abril de 2010.

() Universidade Federal de Lavras/ Pós-Graduação em Fitotecnia, 37200-000 Lavras (MG). E-mail: vanderley-agro@ig.com.br.

$\left(^{(3)}\right.$ Universidade Federal de Lavras, Caixa Postal 3037, 37200-000 Lavras (MG). E-mail: aasoares@ufla.br $\left({ }^{*}\right)$ Autor correspondente.

$\left(^{(4)}\right.$ Empresa de Pesquisa Agropecuária de Minas Gerais, Caixa Postal 176, 37200-000 Lavras (MG). E-mail: moizes@epamig.ufla.br; vanda. cornelio@epamig.ufla.br.

$\left(^{5}\right)$ Embrapa Florestas, Caixa Postal 319, 83411-000 Colombo (PR). E-mail: marcos.deon@ufv.br.

$\left.{ }^{(}\right)$Bolsista de iniciação científica da UFLA. E-mail: alvesnat@gmail.com. 


\section{INTRODUÇÃO}

O perfeito conhecimento da interação genótipos $x$ ambientes é de extrema importância nos programas de melhoramento. É com base nele que se torna possível a seleção de genótipos com adaptação ampla ou específica, a escolha de locais de seleção e a determinação do número ideal de ambientes e de genótipos a serem avaliados durante a seleção (Fox et al., 1997). Assim, inferências e ordenamento sobre os valores genotípicos de tais genótipos são necessários. Considerando que os valores genotípicos são os verdadeiros valores de cultivo e uso (VCU), a análise de dados provenientes de experimentos de VCU em multilocais deve ser realizada sob modelos que ajustem de forma acurada os valores genotípicos livre dos efeitos ambientais, tais como blocos, locais, anos dentre outros.

Os métodos utilizados para estudos da interação genótipos $\mathrm{x}$ ambientes e de adaptabilidade e estabilidade são embasados na análise de variância (Anava), ou seja; sobre médias fenotípicas (BIUDES et al., 2009; SILVA e Duarte, 2006; Morais et al., 2008; Silva et al., 2008). Métodos de estudos de adaptabilidade e estabilidade com base em modelos mistos (REML/BLUP - Restricted Maximum Likelihood/ Best Linear Unbiased Prediction) ainda são raros. Um modelo alternativo com base em modelo misto, para estudos de adaptabilidade e estabilidade, é a Média Harmônica da Performance Relativa dos Valores Genotípicos (MHPRVG), preconizado por RESENDE (2004; 2007a).

O método MHPVG fornece, simultaneamente, a adaptabilidade, a estabilidade e a produtividade em uma mesma medida e na mesma escala do caráter avaliado. Além disso, o modelo ajusta os efeitos de locais e blocos dentro de locais e anos no vetor de efeitos fixos, contemplando dessa forma todos os graus de liberdade disponíveis nas fontes de variação referentes a locais e blocos dentro de locais e anos. Dessa forma, para os valores genotípicos preditos obtidos para um dado genótipo, em cada local, simultaneamente, são utilizados os dados de todos os locais e anos. Nesse caso, de acordo com Resende (2007a), os efeitos aleatórios (genótipos, interação genótipos $x$ locais, interação genótipos $x$ anos, interação genótipos $\mathrm{x}$ locais $\mathrm{x}$ anos) são preditos com maior precisão, uma vez que todo o conjunto de dados é utilizado, bem como os ruídos da interação são eliminados quando se produzem os BLUP's.

BASTOS et al. (2007) utilizaram o método MHPRVG na avaliação da interação genótipos $x$ ambientes em cana-de-açúcar em sete ambientes de Minas Gerais. Neste estudo, os autores destacam, entre outras conclusões que, pelo método MHPRVG, tem-se a vantagem de se interpretar diretamente os valores genéticos para produtividade, adaptabilidade e estabilidade, simultaneamente. Com a cultura do feijoeiro, CARBONELL et al. (2007) estudaram a estabilidade de cultivares e linhagens em diferentes ambientes no Estado de São Paulo, utilizando os métodos MHPRVG, LiN e BINNs (1988) e ANNICCHIARICO (1992), concluindo que os métodos selecionaram praticamente as mesmas linhagens. Contudo, o método MHPRVG seleciona com base em valores genéticos e os demais se baseiam em valores fenotípicos. Na cultura do arroz, ainda não há registro do uso de modelos mistos (REML/BLUP), portanto, é importante realizar trabalhos que possam testar e justificar a utilização desse método.

$\mathrm{Na}$ metodologia de modelos mistos com dados desbalanceados os efeitos do modelo não devem ser testados via teste $\mathrm{F}$, tal como se faz no método da análise de variância (RESENDE, 2007a). Nesse caso, para os efeitos aleatórios, o teste cientificamente recomendado, a fim de verificar a significância dos efeitos do modelo, é o teste da razão de verossimilhança (likelihood ratio test ou LRT) por meio de uma análise de deviance. Esta análise foi sugerida por RESENDE (2007a) e é análoga ao método de NELDER e WEDDERbURN (1972) para variáveis discretas, a qual indica a qualidade do ajuste do modelo. A deviance é uma estatística derivada da razão entre as verossimilhanças do modelo completo, ou modelo saturado, em relação ao modelo sem o efeito que se deseja testar, ou modelo reduzido.

Oobjetivo deste trabalho foi estudar o desempenho genotípico de cultivares e linhagens de arroz de terras altas, avaliadas em Minas Gerais, no período de 1997 / 1998 a $2007 / 2008$, utilizando modelo misto.

\section{MATERIAL E MÉTODOS}

Foram utilizados os resultados de produtividade de grãos obtidos dos ensaios de valor de cultivo e uso (VCU) do programa cooperativo de melhoramento de arroz de terras altas, desenvolvido em Minas Gerais, em parceria com UFLA, EPAMIG, e Embrapa Arroz e Feijão, no período de 1997 / 1998 a 2007/2008, envolvendo 107 cultivares e linhagens, testadas em 11 diferentes locais, ao longo dos 11 anos agrícolas. A relação dos locais por ano agrícola é mostrada na tabela 1 e a de cultivares e linhagens, por ano agrícola, estão apresentadas na tabela 2.

Para as análises estatísticas, utilizou-se o seguinte modelo linear:

$y_{i j k n}=\mu+g_{i}+b_{j k n}+a_{k}+l_{n}+g a_{i k}+g l_{k n}+g a l_{i k n}+g b a l_{i j k n}$

em que: $y_{\text {ijkn }}$ é o valor da observação referente ao tratamento i na repetição $\mathrm{j}$, no ano $\mathrm{k}$ dentro do local $\mathrm{n} ; g_{\mathrm{i}}$ é o efeito do genótipo i; $\mu$ é a média geral; $b_{\mathrm{jkn}}$ é o efeito do bloco $\mathrm{j}$ dentro do ano $\mathrm{k}$ dentro do local $\mathrm{n} ; a_{\mathrm{k}}$ é o efeito do ano de plantio $k$; $l_{\mathrm{n}}$ é o efeito do local $\mathrm{n} ; g a_{\mathrm{ik}}$ 
Tabela 1. Relação dos 11 anos agrícolas e dos 11 locais, onde se realizaram os experimentos

\begin{tabular}{ll}
\hline Ano agrícola & Local dos experimentos \\
\hline $1997 / 98$ & Lambari, Lavras, Patos de Minas \\
1998/99 & Felixlândia, Lambari, Lavras, Patos de Minas, Patrocínio e Uberaba \\
$1999 / 00$ & Felixlândia, Lambari, Lavras, Paracatu, Patos de Minas, Patrocínio, Uberaba \\
$2000 / 01$ & Felixlândia, Lambari, Lavras, Patos de Minas, Patrocínio e Uberaba \\
$2001 / 02$ & Felixlândia, Lambari, Patos de Minas, Uberaba, Uberlândia e Viçosa \\
$2002 / 03$ & Lambari, Lavras, Patos de Minas, Patrocínio, Uberaba, Uberlândia e Viçosa \\
$2003 / 04$ & Felixlândia, Lambari, Patos de Minas, Patrocínio, Piumhi e Viçosa \\
$2004 / 05$ & Felixlândia, Lambari, Lavras, Patos de Minas, Patrocínio e Viçosa \\
$2005 / 06$ & Lambari, Lavras, Patos de Minas, Patrocínio e Piumhi \\
$2006 / 07$ & Lambari, Lavras, Patos de Minas, Patrocínio, Piumhi e São Sebastião do Paraíso \\
$2007 / 08$ & Lambari, Lavras, Patos de Minas, Patrocínio, Piumhi e São Sebastião do Paraíso \\
\hline
\end{tabular}

é o efeito da interação genótipos $\mathrm{x}$ anos de plantio; $g l_{\mathrm{kn}}$ é o efeito da interação genótipos x locais; $g a l_{\mathrm{ikn}}$ é o efeito da interação genótipos $x$ anos $x$ locais; $g b a l_{i \mathrm{jkn}}$ é o erro ou resíduo aleatório.

Na forma matricial, o modelo correspondente é:

$y=X b+Z g+Q g a+T g l+U g l a+e$

Sendo: y o vetor de observações; $b$ o vetor dos efeitos das combinações bloco-local-ano (efeitos fixos do modelo) somados à média geral; $g$ o vetor de efeitos genotípicos, $g$ (assumido como aleatórios); $g a$ o vetor dos efeitos da interação de genótipos $\mathrm{x}$ anos, ga (aleatórios); $g l$ o vetor dos efeitos das interações de genótipos $\mathrm{x}$ locais, $g l$ (aleatório); gla o vetor dos efeitos da interação tripla genótipos $\mathrm{x}$ locais $\mathrm{x}$ anos, gla (assumidos como aleatórios); $e$ o vetor de erros (aleatórios). X, Z, Q, T e U representam as matrizes de incidência para os referidos efeitos respectivamente.

Os efeitos de anos, locais e bloco/anos/locais são agrupados no efeito $b$, pois são efeitos puramente ambientais (RESENDE, 2007a). A estrutura de médias e variâncias é dada conforme RESENDE (2007a) e as equações de modelo misto para o modelo adotado são:

$\left(\begin{array}{c}\hat{b} \\ g \% \\ g a \% \\ g l \% \\ g l a \%\end{array}\right)=\left(\begin{array}{ccccc}X^{\prime} X & X^{\prime} Z & X^{\prime} Q & X^{\prime} T & X^{\prime} U \\ Z^{\prime} X & Z^{\prime} Z+I \lambda_{1} & Z^{\prime} Q & Z^{\prime} T & Z^{\prime} U \\ Q^{\prime} X & Q^{\prime} Z & Q^{\prime} Q^{\prime} I \lambda_{2} & Q^{\prime} T & Z^{\prime} U \\ T^{\prime} X & T^{\prime} Z & T^{\prime} Q & T^{\prime} T+I \lambda_{3} & T^{\prime} U \\ U^{\prime} X & U^{\prime} Z & U^{\prime} Q & U^{\prime} T & U^{\prime} U+I \lambda_{4}\end{array}\right)^{-1}\left(\begin{array}{c}X^{\prime} y \\ Z^{\prime} y \\ Q^{\prime} y \\ T^{\prime} y \\ U^{\prime} y\end{array}\right)$

Sendo:

$$
\begin{aligned}
& \lambda_{1}=\frac{\sigma_{e}^{2}}{\sigma_{g}^{2}}=\frac{1-h_{g}^{2}-c_{g a}^{2}-c_{g l}^{2}-c_{g l a}^{2}}{h_{g}^{2}}, \\
& \lambda_{2}=\frac{\sigma_{e}^{2}}{\sigma_{g a}^{2}}=\frac{1-h_{g}^{2}-c_{g a}^{2}-c_{g l}^{2}-c_{g l a}^{2}}{c_{g a}^{2}},
\end{aligned}
$$

$\lambda_{3}=\frac{\sigma_{e}^{2}}{\sigma_{g l}^{2}}=\frac{1-h_{g}^{2}-c_{g a}^{2}-c_{g l}^{2}-c_{g l a}^{2}}{c_{g l}^{2}}$,

$\lambda_{4}=\frac{\sigma_{e}^{2}}{\sigma_{g l a}^{2}}=\frac{1-h_{g}^{2}-c_{g a}^{2}-c_{g l}^{2}-c_{g l a}^{2}}{c_{g l a}^{2}} \mathrm{e} h_{g}^{2}$ é a herdabilidade no sentido amplo e $c^{2}$ corresponde a coeficientes de determinação de cada efeito respectivo.

Os valores da média harmônica dos valores genotípicos (MHVG) para a avaliação da estabilidade, da performance relativa dos valores genotípicos (PRVG) para a adaptabilidade e da média harmônica da performance relativa dos valores genotípicos (MHPRVG) para a estabilidade, a adaptabilidade e a produtividade, simultaneamente, para todos os genótipos foram obtidos conforme as expressões:

$$
\begin{aligned}
& M H V G=\frac{l}{\sum_{i=1}^{l} \frac{1}{V G_{j}}}, P R V G=\frac{1}{l}\left(\frac{\sum V G_{j}}{M_{j}}\right) \mathrm{e} \\
& M H P R V G=\frac{l}{\sum_{l=1}^{l} \frac{1}{P R V G_{j}}}
\end{aligned}
$$

em que $l$ : número de locais; VG: valor genotípico; $j$ : genótipos.

A significância dos efeitos do modelo foi estimada pela análise de deviance, conforme recomendações de RESENDE (2007a). As deviances foram obtidas por meio de análises com e sem os efeitos de $g, g a$, gl e gla. Em seguida, subtraiu-se de cada deviance do modelo completo a deviance sem o referido efeito, e confrontando-o com o valor do qui-quadrado com um grau de liberdade, 


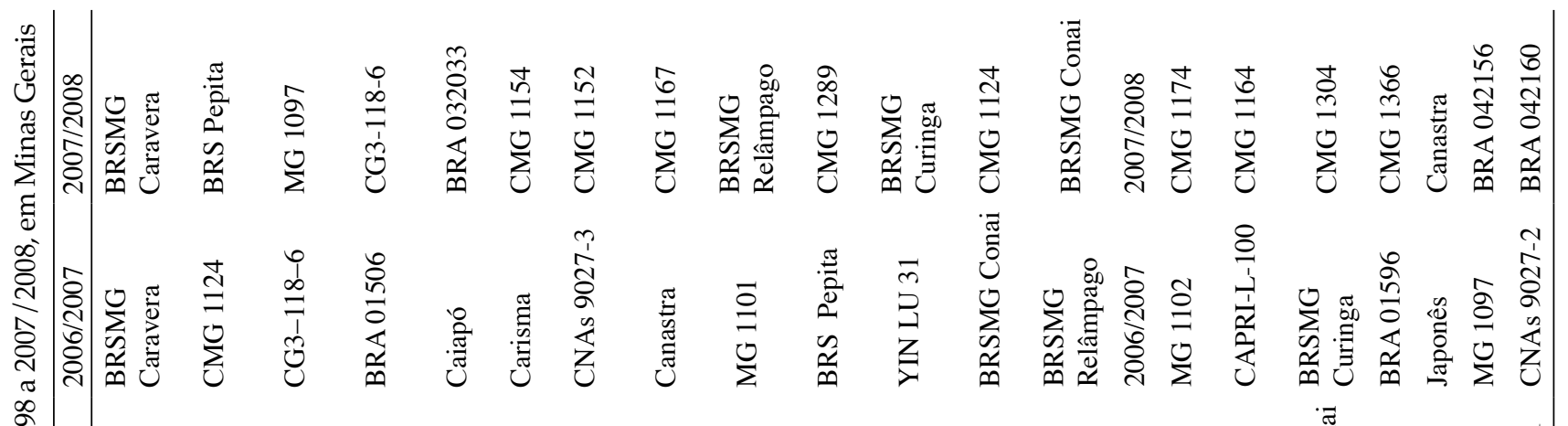
s.

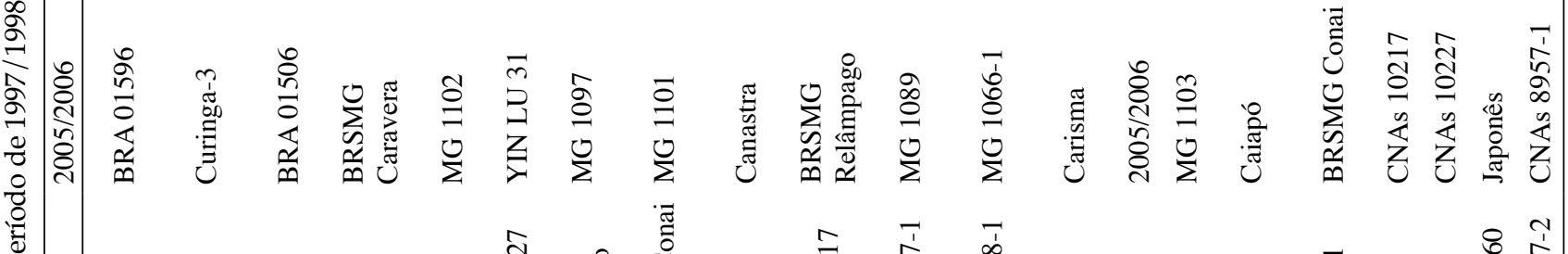

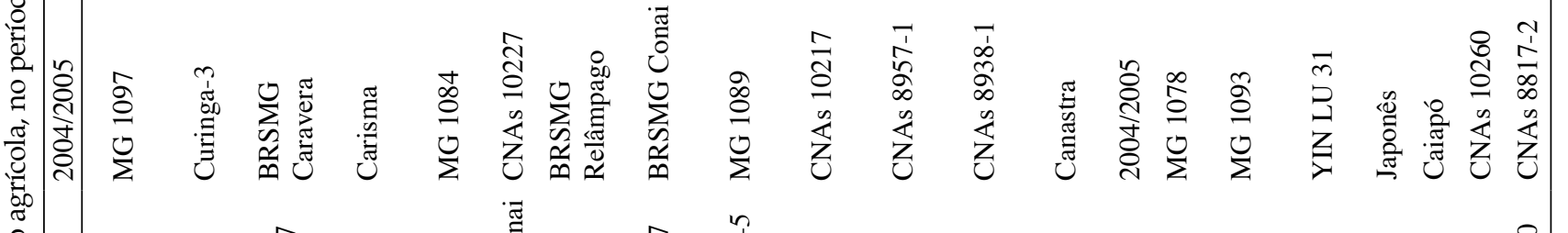

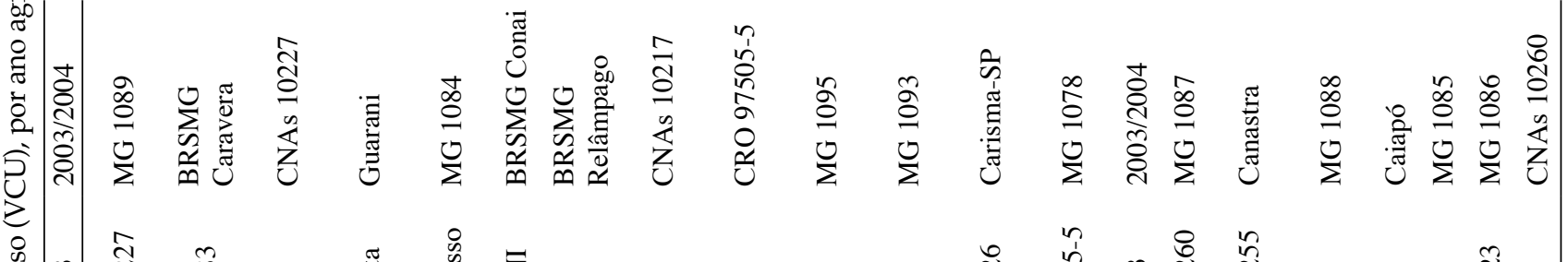

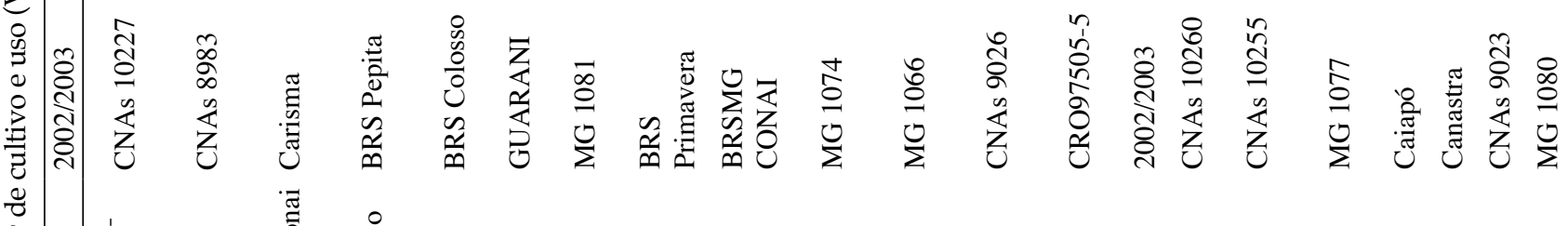

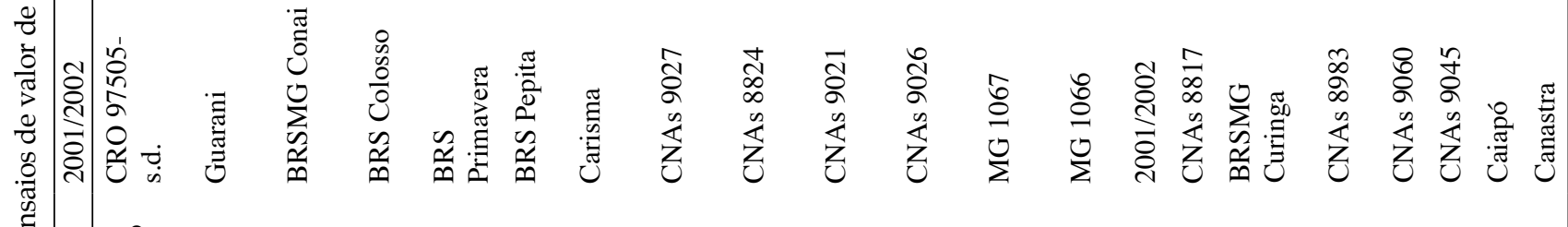

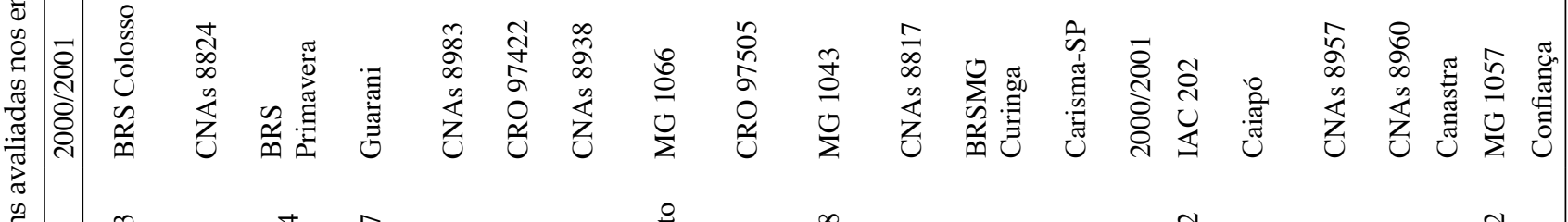

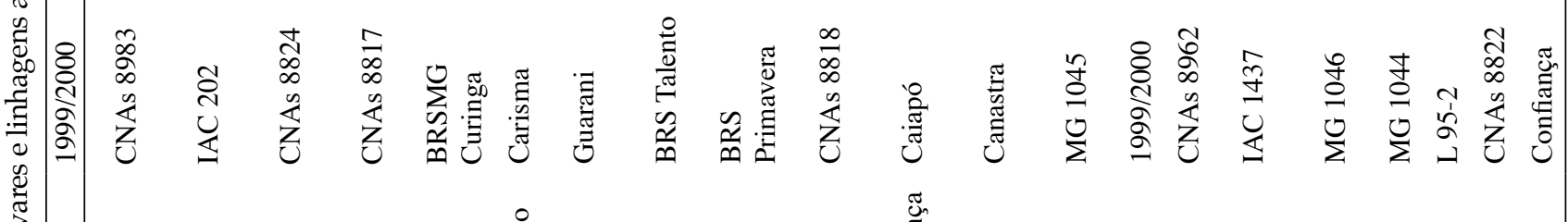

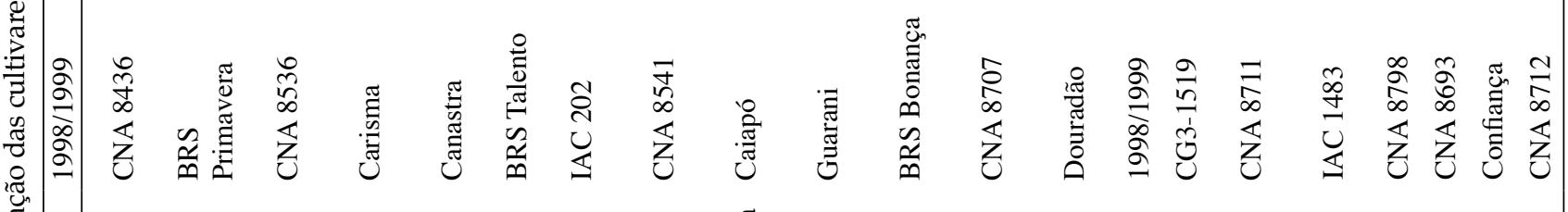

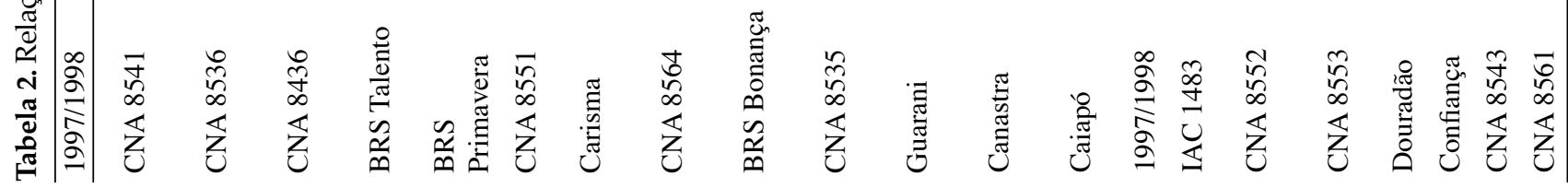


a $1 \%$ e $5 \%$ de probabilidade. Matematicamente: $L R T=-2 \ln \left(\frac{M V \text { do } \text { modelo reduzido }}{M V \text { do modelo completo }}\right)$, sendo $\ln$ o logaritmo neperiano e MV máxima verossimilhança.

As análises foram realizadas com o aplicativo SELEGEN REML / BLUP (RESENDE, 2007b).

\section{RESULTADOS E DISCUSSÃO}

$O$ resultado da análise de deviance para efeitos de genótipos e os efeitos das interações genótipos $\mathrm{x}$ locais, genótipos $\mathrm{x}$ anos e genótipos $\mathrm{x}$ locais $\mathrm{x}$ anos, seus respectivos componentes de variância e coeficientes de determinação na análise conjunta dos 11 locais e 11 anos, é apresentado na tabela 3. Verificou-se, pela análise de deviance, que os efeitos de genótipos, das interações genótipos $\mathrm{x}$ local e genótipos $\mathrm{x}$ anos, bem como seus componentes de variância $(V g, V g l$ e $V g a)$ e coeficientes de determinação $\left(h^{2}, c_{g l}^{2}\right.$ e $\left.c_{g a}^{2}\right)$ dos respectivos efeitos, foram estatisticamente significativos $(\mathrm{p} \leq 0,05)$. Portanto, apenas o efeito da interação tripla genótipos $\mathrm{x}$ locais $\mathrm{x}$ anos, assim como seus respectivos componentes de variância $(V g l a)$ e coeficientes de determinação $\left(c_{g l a}^{2}\right)$ não o foram. A análise de deviance evidenciou, assim, a presença de variabilidade genética entre as linhagens testadas e interação de locais e anos com cada genótipo individualmente.

Aтroch et al. (2000), que estudaram a adaptabilidade e estabilidade fenotípicas de nove linhagens de arroz de terras altas testadas em Minas Gerais em quatro locais e três anos, identificaram interação significativa para genótipos x locais, genótipos $x$ anos e genótipos $x$ locais $x$ anos. Ainda, conforme os autores, a magnitude da interação genótipos $x$ locais (de $63.155,000)$ foi mais expressiva que a interação genótipos $x$ anos (de 22.728,000), sugerindo que se deveriam testar as linhagens, como forma de otimizar o programa de melhoramento de arroz de terras altas, em um número maior de locais do que de anos. No presente estudo, a magnitude da interação $V g l$ foi ligeiramente superior a $V g a$ (Tabela 3), corroborando com os resultados de Aтroch et al. (2000). Assim, o comportamento dos genótipos nesse estudo foi semelhante ao avaliado por esses autores, à exceção apenas aos efeitos da interação tripla genótipos $\mathrm{x}$ locais $\mathrm{x}$ anos. Todavia, pelos valores genotípicos, as diferenças entre os efeitos das interações de genótipos x locais (deviance de 19.444,21) e genótipos $x$ anos (deviance de 19.443,79) foram de baixa magnitude e próximas entre si.

Os coeficientes de determinação $\left(c_{g l}^{2}\right.$ para a interação genótipos $\mathrm{x}$ locais; $c_{g a}^{2}$ para a interação genótipos $\mathrm{x}$ anos e $c_{g l a}^{2}$ para a interação genótipos $x$ locais $x$ anos) indicam o quanto cada componente contribui para a variância fenotípica total. Dessa forma, as interações genótipos $\mathrm{x}$ locais; genótipos $\mathrm{x}$ anos $\mathrm{e}$ genótipos $x$ locais $x$ anos contribuíram com 5,08\%, 4,68\% e $1,9 \%$ respectivamente.

\section{Estabilidade dos valores genotípicos / MHVG}

A MHVG - média harmônica dos valores genotípicos - computa a estabilidade e a produtividade de grãos, simultaneamente. Assim, a seleção baseada na MHVG contempla esses dois atributos conjuntamente. ComoaMHVGpenalizaainstabilidade, quandogenótipos são avaliados em diversos locais, o resultado é que a nova média obtida é ajustada por essa penalização.

O resultado da avaliação da $\mathrm{MHVG}$ para as 25 cultivares e linhagens superiores das 107 testadas nos 11 locais e 11 anos é relatado na tabela 4. Como mencionado, os valores da MHVG são os próprios valores da produtividade de grãos, penalizados pela instabilidade, o que certamente facilita a seleção das linhagens produtivas e ao mesmo tempo mais estáveis. A instabilidade climática e a heterogeneidade dos solos são maiores nas condições tropicais. Esta condição exige que as cultivares recomendadas aos agricultores devam aliar produtividade de grãos e maior estabilidade. Portanto, o critério MHVG atende exatamente essas duas premissas que a cultivar deve apresentar.

Tabela 3. Deviance, componentes de variância e coeficientes de determinação referentes à análise conjunta global envolvendo 107 cultivares e linhagens testadas em 11 locais e 11 anos. 1997/1998 a 2007/ 2008

\begin{tabular}{lcccc}
\hline Efeito & Deviance & LRT $\left(\chi^{2}\right)$ & Componentes de variância & Coeficientes de determinação \\
\hline Genótipos & $19453,87^{+}$ & $15,20^{* *}$ & $V g=57742,19$ & $h^{2} g=0,097$ \\
Genótipos x locais & $19444,21^{+}$ & $5,54^{*}$ & $V g l=30266,24$ & $c^{2} g l=0,0508$ \\
Genótipos x anos & $19443,79^{+}$ & $5,12^{*}$ & $V g a=27878,57$ & $c^{2} g a=0,0467$ \\
Genótipos x locais x anos & $19438,58^{+}$ & $0,08^{\mathrm{ns}}$ & $V g l a=11352,16^{\mathrm{ns}}$ & $c^{2} g l a=0,02^{\mathrm{ns}}$ \\
Resíduo & & - & $V e=468824,32$ & $c^{2}$ res $=0,84$ \\
\hline Modelo Completo & 19438,67 & - & - & $c^{2}$ total $=1,00$
\end{tabular}

${ }^{+}$: Deviance do modelo ajustado sem os efeitos correspondente. * $\mathrm{e}^{* *}$ : Significativo pelo teste qui-quadrado a $5 \%(3,84)$ e $1 \%(6,63)$ respectivamente. 
Tabela 4. Estabilidade dos valores genotípicos (MHVG), adaptabilidade dos valores genotípicos (PRVG), valores genotípicos médios capitalizado pela interação (PRVG* $\mathrm{MG}$ ), estabilidade e adaptabilidade de valores genotípicos (MHPRVG) e valores genotípicos médios nos locais (MHPRVG*MG) para produção de grãos de cultivares e linhagens de arroz avaliadas em Minas Gerais, no período de 1997 / 1998 a 2007 / 2008

\begin{tabular}{|c|c|c|c|c|c|c|c|}
\hline Genótipo & $\mathrm{MHVG}^{1}$ & Genótipo & PRVG & $\mathrm{PRVG}^{*} \mathrm{MG}^{\mathrm{a}}$ & Genótipo & MHPRVG & MHPRVG*MG \\
\hline Curinga-3 & 3965,7404 & BRSMG Caravera & 1,1583 & 4079,2178 & BRSMG Caravera & 1,1575 & 4076,3951 \\
\hline CNA8983 & 3935,5052 & Curinga-3 & 1,1346 & 3995,6427 & Curinga-3 & 1,1342 & 3994,3049 \\
\hline Guarani & 3932,2042 & MG1089 & 1,0842 & 3818,1402 & MG1089 & 1,0834 & 3815,3367 \\
\hline BRSMG Caravera & 3925,6388 & MG1097 & 1,0812 & 3807,8344 & MG1097 & 1,0807 & 3806,0925 \\
\hline CNA8824 & 3898,9942 & CNA8436 & 1,0784 & 3797,9539 & CNA8436 & 1,0782 & 3796,9495 \\
\hline BRS Primavera & 3892,9927 & BRA01506 & 1,0734 & 3780,1209 & BRA01506 & 1,0732 & 3779,4792 \\
\hline IAC 202 & 3881,9464 & BRA01596 & 1,0650 & 3750,6794 & BRA0151596 & 1,0648 & 3749,9076 \\
\hline MG 1044 & 3847,5332 & CNA8983 & 1,0619 & 3739,5941 & CNA8983 & 1,0614 & 3738,0063 \\
\hline BRS Talento & 3831,1953 & Guarani & 1,0617 & 3738,8621 & Guarani & 1,0609 & 3736,0658 \\
\hline Carisma & 3791,9405 & CNA8536 & 1,0609 & 3736,1965 & CNA8536 & 1,0607 & 3735,6471 \\
\hline MG 1089 & 3784,6090 & BRS Colosso & 1,0568 & 3721,7246 & BRS Colosso & 1,0563 & 3720,0135 \\
\hline CNA 8436 & 3776,5669 & Carisma & 1,0565 & 3720,5658 & Carisma & 1,0562 & 3719,5867 \\
\hline BRS Colosso & 3766,6489 & CNA 8541 & 1,0553 & 3716,6242 & CNA 8541 & 1,0552 & 3716,1395 \\
\hline CRO 97505 & 3753,6273 & CRO 97505 & 1,0532 & 3709,1384 & CRO 97505 & 1,0531 & 3708,8900 \\
\hline CNAs 8817 & 3753,3675 & MG1084 & 1,0525 & 3706,7537 & MG1084 & 1,0523 & 3705,7963 \\
\hline CNAs 10227 & 3752,0841 & CG3-118-6 & 1,0519 & 3704,4213 & CG3-118-6 & 1,0518 & 3704,0213 \\
\hline CNA 8536 & 3714,4728 & CNA10227 & 1,0519 & 3704,4102 & CNA10227 & 1,0517 & 3703,7696 \\
\hline CNA 8541 & 3695,5609 & CNA8824 & 1,0518 & 3704,3109 & CNA8824 & 1,0517 & 3703,6673 \\
\hline Genótipo & $\mathrm{MHVG}^{1}$ & Genótipo & PRVG & $\mathrm{PRVG}^{*} \mathrm{MG}^{*}$ & Genótipo & MHPRVG & MHPRVG*MG \\
\hline MG 1081 & 3693,0454 & IAC202 & 1,0518 & 3704,0770 & IAC202 & 1,0514 & 3702,9104 \\
\hline MG 1084 & 3676,6225 & BRS Primavera & 1,0501 & 3698,2976 & BRS Primavera & 1,0499 & 3697,3111 \\
\hline MG 1097 & 3657,6763 & BRS Pepita & 1,0447 & 3679,2935 & BRS Pepita & 1,0446 & 3678,8670 \\
\hline CRO 97595-5 & 3645,5295 & BRSMG Conai & 1,0436 & 3675,3468 & BRSMG Conai & 1,0436 & 3675,1000 \\
\hline BRS Pepita & 3625,9889 & MG1044 & 1,0428 & 3672,6262 & MG1044 & 1,0421 & 3670,1552 \\
\hline BRSMG Conai & 3622,2405 & BRSMG Relâmpago & 1,0420 & 3669,7384 & BRSMG Relâmpago & 1,0418 & 3668,9247 \\
\hline CNAs 8818 & 3615,2055 & BRS Talento & 1,0391 & 3659,4915 & BRS Talento & 1,0389 & 3658,6243 \\
\hline
\end{tabular}

a: Média geral do experimento.

Pelos resultados da MHVG, verificou-se que, dentre as cultivares comerciais, as que melhor associam produtividade com estabilidade, em ordem decrescente, são: a Guarani, a BRSMG Caravera, a BRS Primavera, a IAC 202, a BRS Talento, a Carisma, a BRS Colosso, a BRS Pepita e a BRSMG Conai. A linhagem MG 1097, em vias de lançamento, também, se destacou nesse quesito. Entre as cultivares atualmente mais aceitas no mercado, destacam-se a BRSMG Caravera e a BRS Primavera (um dos genitores da BRSMG Caravera) com bom desempenho em diferentes condições ambientais, e boa estabilidade.

A BRSMG Caravera, a BRS Primavera e outras cultivares relacionadas pela MHVG, atenderam também ao critério de CRUz et al. (1989), quando afirmam que o genótipo de comportamento ideal deve possuir elevada média de produtividade de grãos e baixa sensibilidade às mudanças de ambiente. ScAPIM et al. (2000) também ressaltaram que a maior estabilidade está associada, obrigatoriamente, à maior produtividade.

SOARES et al. (2007), em estudo de estabilidade dos ensaios dos VCU do ano 2004/2005 em dez ambientes (locais) pelos métodos de LiN e Binns (1988) e ANNICCHIARICO (1992), identificaram a linhagem MG 1097 como a mais produtiva e a mais estável, seguida da linhagem Curinga-3. Este estudo corrobora o ótimo desempenho da linhagem Curinga-3, primeira classificada pelo ordenamento MHVG. Por outro lado, no presente trabalho, a linhagem MG 1097 classificou-se apenas na 21. a posição. Os mesmos autores, estudando a estabilidade das 13 cultivares e linhagens comuns aos anos agrícolas 2003/2004 e 2004/2005 identificaram a 
BRSMG Caravera como a produtiva e a mais estável, seguida da linhagem MG 1089.

É natural e esperado que a utilização de diferentes métodos de estudo de estabilidade, como também a quantidade de locais, altere a classificação dos genótipos em estudo (Vencovsky e BARRIGA, 2002; CRUZ et al., 2004). Entretanto, o método MHVG por ser obtido com base em REML/BLUP e ser trabalhado com a recíproca dos valores fenotípicos $(1 / y)$, conduz a resultados que é função da média harmônica dos dados (1/H) (RESENDE, 2004). Dessa forma quanto menor o desvio-padrão do desempenho genotípico através dos locais, maior será a média harmônica de seus valores genotípicos. Além disso, a MHVG penaliza os valores genotípicos de cada genótipo pela instabilidade (RESENDE, 2007a), o que garante maior precisão e acurácia no ordenamento dos genótipos dentro e dentre locais.

\section{Adaptabilidade de valores genotípicos / PRVG}

Adaptabilidade é a capacidade de as linhagens serem responsivas de forma vantajosa à melhoria do ambiente (MARIOTTI et al., 1976), portanto, essa é uma característica, de grande valor e procurada pelos fitomelhoristas para as novas cultivares. Para identificar essa característica, é necessário utilizar métodos apropriados e, dentre os existentes, está a performance relativa dos valores genotípicos (PRVG) que capitaliza a capacidade de resposta de cada linhagem à melhoria do ambiente. Os resultados desse estudo estão apresentados na tabela 4 para as 25 cultivares e linhagens superiores para essa característica. Utilizando-se o produto da PRVG pela média geral $\left(3706,26 \mathrm{~kg} \mathrm{ha}^{-1}\right)$, obteve-se a coluna PRVG x MG para os 25 genótipos superiores, valor genotípico médio, capitalizado pela interação.

Observando os valores da PRVG x MG, constatouse que a cultivar BRSMG Caravera, no conjunto das 107 cultivares e linhagens avaliadas, foi a de maior adaptabilidade genotípica associada à produtividade de grãos, respondendo, assim, com grande vantagem à melhoria dos ambientes. Destacaram-se também, dentre as cultivares comerciais, em ordem decrescente, a Guarani, a Carisma, a BRS Colosso, a IAC 202, a BRS Primavera, a BRS Pepita, a BRSMG Conai, a BRSMG Relâmpago e a BRS Talento. Por coincidência, à exceção da BRSMG Relâmpago, todas as outras sete cultivares estão entre as 25 de maior estabilidade, agregando adaptabilidade e estabilidade genotípicas. A linhagem MG 1097, também exibe excelente adaptabilidade genotípica, ocupando a quarta posição (Tabela 4). Esse estudo confirmou a boa performance das cultivares lançadas e, ou recomendadas para Minas Gerais nos quesitos adaptabilidade e estabilidade genotípicas, dando segurança aos agricultores no plantio dessas cultivares.

\section{Estabilidade e adaptabilidade de valores genotípicos / MHPRVG}

O método da média harmônica da performance relativa dos valores genotípicos (MHPRVG), que se baseia em valores genotípicos preditos, via modelos mistos, agrupa em uma única estatística, a estabilidade, a adaptabilidade e a produtividade, facilitando, de modo singular, a seleção de genótipos superiores. A MHPRVG das 25 cultivares e linhagens superiores, dentre as 107 avaliadas, nos 11 anos e 11 locais, bem como o produto da MHPRVG pela média geral $(3706,26$ $\mathrm{kg} \mathrm{ha}^{-1}$ ), são apresentados na tabela 4. A MHPRVG*MG fornece os valores genotípicos de cada cultivar e/ou linhagem penalizados pela instabilidade e capitalizados pela adaptabilidade. Assim, a tabela 4 agrupa em uma única estatística, além da MHVG e PRVG, a estabilidade, a adaptabilidade e a produtividade de grãos, simultaneamente, das 25 melhores cultivares e linhagens.

RESENDE (2004) comparou essa metodologia com o de Lin e BinNs (1988) e o de ANNicCHIARICo (1992), utilizando valores genotípicos e observou que elas fornecem resultados análogos. Entretanto, a MHPRVG, além de ser estimada por REML/BLUP, fornece os resultados na própria escala de avaliação do caráter.

As cinco melhores linhagens, considerando todos os ambientes deste estudo, com base no método MHPRVG, foram: BRSMG Caravera, Curinga-3, MG 1089, MG 1097 e CNA 8436. Assim, a BRSMG Caravera respondeu, em média, 1,16 vezes a média dos locais em que foi cultivada, já, a Curinga-3 respondeu 1,13 vezes, e assim sucessivamente para as demais linhagens.

As outras cultivares comerciais que se destacaram entre as 25 melhores, nos quesitos produtividade de grãos, estabilidade e adaptabilidade, simultaneamente, foram a Guarani, a BRS Colosso, a Carisma, a IAC 202, a BRS Primavera, a BRS Pepita, a BRSMG Conai, a BRSMG Relâmpago e a BRS Talento. Destas, estão em desuso ou muito pouco plantadas, a Guarani, a BRS Colosso e a IAC 202, sendo a Guarani por problemas de qualidade de grãos e as outras duas pela alta suscetibilidade a brusone da panícula. As linhagens MG 1097 e CG3 118-6 se destacaram no tocante às três características, conjuntamente, ocupando a quarta e décima sexta posições, respectivamente, no ordenamento pela MHPRVG.

Vale ressaltar que a linhagem CNA 8436, que não foi lançada como cultivar, foi avaliada somente em 1997/ 1998 e 1998/1999, no entanto, aparece como uma das mais estáveis, adaptadas e produtivas (quinto no ordenamento pela MHPRVG, com produtividade de $\left.3796,95 \mathrm{~kg} \mathrm{ha}^{-1}\right)$. Essa alta estabilidade e adaptabilidade 
podem estar superestimadas, pois tal linhagem foi avaliada em pequeno número de ambientes.

Observando-se as cinco cultivares e linhagens seguintes, em destaque na tabela 4, para MHPRVG (BRA 015506, BRA 01596, CNAs 8983, Guarani e CNA 8536) é pertinente salientar que o comportamento delas são semelhantes entre si e muito próximos às cultivares e linhagens ordenadas na terceira, quarta e quinta posições e todas responderam de 1,06 a 1,08 vezes a média dos locais onde foram cultivadas.

Estudos utilizando o método MHPRVG foram realizados em outras espécies. Em cana-de-açúcar, para o caráter tonelada de cana por hectare (TCH), OLIVEIRA et al. (2005) observaram valores para MHPRVG igual a 1,28 e 1,19 para os clones ordenados em primeiro e segundo lugares respectivamente. Nesse estudo os clones foram avaliados em três locais em um único ano. No trabalho de BAstos et al. (2007), também com cana-de-açúcar, os valores para MHPRVG do primeiro e segundo clones, no ordenamento geral, foram de 1,21 e 1,17, respectivamente. Com feijão, CARBOnEll et al. (2007) relataram valores de MHPRVG iguais a 1,11 e 1,10 para o primeiro e segundo genótipo, respectivamente, valores esses relativamente próximos aos do presente trabalho (1,16 e 1,13 respectivamente).

Em síntese, em BRSMG Caravera e Curinga-3 houve superioridade média de $15,7 \%$ e $13,8 \%$ respectivamente, sobre a média geral dos 107 genótipos, nos 11 locais e nos 11 anos. Já, a MG 1089 teve superioridade de apenas $8 \%$, e assim sucessivamente (Tabela 4). Portanto, a BRSMG Caravera e a Curinga-3 foram bem superiores às demais, pela MHPRVG, de forma simultânea, para adaptabilidade, estabilidade e produtividade, confirmando seus desempenhos nas análises MHVG e PRVG. Das cinco melhores cultivares e linhagens selecionadas pela MHVG, apenas a BRSMG Caravera e a Curinga-3 foram coincidentes para PRVG e MHPRVG. O único material que esteve em primeira ou segunda posição no ordenamento pelos três métodos, simultaneamente, foi a Curinga-3

Nenhuma das linhagens que entrou no último ano da análise (2007/2008) está entre as 25 superiores, o que de certa forma, já era esperado, uma vez que pelo critério do método REML/BLUP materiais que aparecem menos nas avaliações são mais penalizados pelo efeito "shrinkage". As predições de pequenas amostras (tratamentos) são mais penalizadas, ocasionando redução de seus valores.

\section{CONCLUSÕES}

1. As linhagens de arroz de terras altas desenvolvidas para plantio em Minas Gerais apresentam ótima performance para estabilidade, adaptabilidade e produtividade grãos, simultaneamente.

2. A BRSMG Caravera, entre todas as cultivares e linhagens avaliadas de 1997 a 2008, foi a de melhor desempenho pela MHPRVG, ou seja, maior estabilidade, adaptabilidade e produtividade de grãos, simultaneamente, proporcionando aos orizicultores de Minas Gerais alta segurança no seu cultivo.

3. A metodologia de modelos mistos é uma estatística de fácil aplicação e de grande utilidade na avaliação de ensaios de valor de cultivo e uso, sobretudo na seleção e no descarte de linhagens a cada ano agrícola.

\section{AGRADECIMENTOS}

À Fapemig, ao CNPq, à UFLA, à Epamig e a Embrapa Arroz e Feijão, que possibilitaram o desenvolvimento deste trabalho.

\section{REFERÊNCIAS}

ANNICCHIARICO, P. Cultivar adaptation and recommendation from alfalfa trials in northern Italy. Journal of Genetics and Plant Breeding, v.46, p.269-278, 1992.

ATROCH, A.L.; SOARES, A.A.; RAMALHO, M.A.P. Adaptabilidade e estabilidade de linhagens de arroz de sequeiro testadas no Estado de Minas Gerais. Ciência e Agrotecnologia, v. 24, p.541-548, 2000.

BASTOS, I.T.; BARBOSA, M.H.P.; RESENDE, M.D.V.; PETERNELLI, L.A.; SILVEIRA, L.C.I.; DONDA, L.R.; FORTUNATO, A.A.; COSTA, P.M.A.; FIGUEIREDO, I.C.R. Avaliação da interação genótipo $x$ ambiente em cana-deaçúcar via modelos mistos. Pesquisa Agropecuária Tropical, v.37, p.195-203, 2007.

BIUDES, G.B; CAMARGO, C.D.O; FERREIRA FILHO, A.W.P.; PETTINELLI JUNIOR, A.; FOLTRAN, D.E.; CASTRO, J.L.; AZEVEDO FILHO, J.A. Adaptabilidade e estabilidade de linhagens de diaplóides de trigo. Bragantia, v.68, p.63-74, 2009.

CARBONELL, S.A.M.; CHIORATO, A.F.; RESENDE, M.D.V.; DIAS, L.A.S.; BERALDO, A L.A.; PERINA, E.F. Estabilidade de cultivares e linhagens de feijoeiro em diferentes ambientes no estado de São Paulo. Bragantia, v.66, p.193-201, 2007.

CRUZ, C.D.; REGAZZI, A.J.; CARNEIRO, P.C.S. Modelos biométricos aplicados ao melhoramento genético. vol.1, 3.ed. Viçosa: UFV, 2004. 480p.

CRUZ, C.D.; TORRES, R.A.; VENCOVSKY, R. An alternative approach to the stability analysis proposed by Silva and Barreto. Revista Brasileira de Genética, v.12, p.567-580, 1989. 
FOX, P.N.; CROSSA, J.; ROMAGOSA, I. Multi-environment testing and genotype-environment interaction. In: KEMPTON, R.A.; FOX, P.N. (Ed.). Statistical methods for plant variety evaluation. New York: Chapman \& Hall, 1997. p. 117-138.

LIN, C. S.; BINNS, M. R. A superiority measure of cultivar performance for cultivar $\mathrm{x}$ location data. Canadian Journal of Plant Science, v. 68, p. 193-198, 1988.

MARIOTTI, J.A.; OYARZABAL, E.S.; OSA, J.M.; BULACIO, A.N.R.;ALMADA, G.H. Analisis deestabilidad y adaptabilidad de genotipos de caña de azucar. Revista Agronomica del Noroeste Argentino, v.13, p.105-27, 1976.

MORAIS, L.K.; MOURA, M.F.; VENCOVSKY, R.; PINHEIRO, J.B. Adaptabilidade e estabilidade fenotípica em soja avaliada pelo método de Toler. Bragantia, v.67, p. 275-284, 2008.

NELDER, J.A; WEDDERBURN, R.W.M. Generalized linear model. Journal Royal Statistic Society, v.135, p.370-384, 1972.

OLIVEIRA, R.A.; RESENDE, M.D.V.; DAROS, E.;BESPALHOK FILHO, J.C.; ZAMBON, J.L. C.; IDO, O.T.; WEBER, H.; KOEHLER, H.S. Genotypic evaluation and selection of sugarcane clones in three environments in the state of Paraná. Crop Breeding and Applied Biotechnology, v.5, p.426-434, 2005.

RESENDE, M.D.V. Matemática e estatística na análise de experimentos e no melhoramento genético. Colombo: Embrapa Florestas, 2007a. 561p.

RESENDE, M. D. V. SELEGEN-REML/BLUP: sistema estatístico e seleção genéticacomputadorizada via modelos lineares mistos. Colombo: Embrapa Florestas, 2007b. 361p.
RESENDE, M.D.V. Métodos estatísticos ótimos na análise de experimentos de campo. Colombo: Embrapa Florestas, 2004. 65p. (Documentos 100)

RESENDE, M.D.V. Genética biométrica e estatística no melhoramento de plantas perenes. Brasília: Embrapa Informação Tecnológica, 2002. 975p.

RESENDE, M.D.V.; DUARTE, J.B. Precisão e controle de qualidade em experimentos de avaliação de cultivares. Pesquisa Agropecuária Tropical, v.37, p.182-194, 2007.

SCAPIM, C.A.; OLIVEIRA, V.R.; BRACCINI, A.L.; CRUZ, C.D.; ANDRADE, C.A.; VIDIGAL, M.C.G. Yield stability im maize (Zea mays L.) and correlation among the parameters of the Eberhart and Russel; Lin and BINNS and Hühn models. Genetics and Molecular Biology, v.23, p.387-393, 2000.

SILVA, W.C.J.; DUARTE, J.B. Métodos estatísticos para estudo de adaptabilidade e estabilidade fenotípica em soja. Pesquisa Agropecuária Brasileira, v.41, p.23-30, 2006.

SILVA, F.L.; SOARES, P.C.; CARGNIN, A.; SOUZA, M.A.; SOARES, A.A.; CORNÉLIO, V.M.O.; REIS, M.S. Methods of adaptability and stability analysis in irrigated rice genotypes in Minas Gerais, Brazil. Crop Breeding and Applied Biotechnology, v.8, p.119-126, 2008.

SOARES, A.A.; REIS, M.S; CORNÉLIO, V.M.O; SOARES, P.C.; VIEIRA, A.R.; SOUZA, M.A. Stability of upland rice lines in Minas Gerais, Brazil. Crop Breeding and Applied Biotechnology, v.7, p.394-398, 2007.

VENCOVSKY, R.; BARRIGA, P. Genética biométrica no fitomelhoramento. Ribeirão Preto: Sociedade Brasileira de Genética, 1992. 496p. 
